\title{
A PESQUISA EM ADMINISTRAÇÃO ESTRATÉGICA: UM ESTUDO BIBLIOMÉTRICO EM PERIÓDICOS INTERNACIONAIS DE ESTRATÉGIA NO PERÍODO DE 2008 A 2013
}

\section{RESUMO}

Neste estudo analisamos o campo científico da estratégia por meio de uma leitura sobre as produções científicas publicadas em periódicos internacionais de estratégia com alto fator de impacto, nos últimos cinco anos. A natureza desse trabalho foi viabilizada por meio do método bibliométrico, pela análise de citação e cocitações, sendo utilizada conjuntamente a análise fatorial, que nos permitiu levantarmos as principais tendências teóricas desenvolvidas dentro do campo da estratégia, bem como a conexão existente entre os principais autores. Os dados coletados a partir do método bibliométrico elucidaram as abordagens teóricas utilizadas: a Resource Based View - RBV, Inovação, Conhecimento, Aprendizagem e Capability. Os resultados confirmaram a tendência apontada por estudos anteriores da emergência da RBV, pois foi identificada a predominância também dessa teoria nos trabalhos aqui analisados, bem como sua relação com conhecimento, aprendizagem organizacional, e com inovação. Portanto esse estudo contribui para ampliar o entendimento das escolas de pensamento que atualmente permeiam o campo da estratégia, para que assim possam orientar futuros estudos nesse campo com base nas lacunas conceituais identificadas.

Palavras Chave: Estratégia. Bibliometria. Análise de citação e cocitações. Visão Baseada em Recursos.

\section{ABSTRACT}

In this study we analyzed the scientific field of strategy through a research of the academic production published in international top-ranked strategy journals in the last five years. This work was made possible through the bibliometric method, using citation and co-citations analysis, and jointly with the use of factor analysis to rise up the main theoretical trends developed within the field of strategy, and the connection between the main authors. The data collected through the bibliometric tools allowed us to identify the most used theoretical approaches: the Resource Based View - RBV, Innovation, Knowledge, Learning and Capability. The results confirm the trend reported by previous studies about the emergence of RBV, since it was identified the predominance of this theory in the works analyzed here, as well as its relationship with knowledge, organizational learning, and innovation. Therefore this study contributes to extend the understanding of the main theoretical approaches that currently permeate the field of strategy, so that they can guide future studies in this field based on the identified conceptual gaps.

Keywords: Strategy. Bibliometric study. Citation and co-citation analysis. Resource-based view.

Rosiele Fernandes Pinto ${ }^{1}$ Luiz Antonio de Camargo Guerrazzi ${ }^{2}$ Bernardo Paraiso de Campos Serra ${ }^{3}$ Claudia Terezinha Kniess ${ }^{4}$

\footnotetext{
${ }^{1}$ Universidade Federal da Paraíba - UFPB, Brasil

E-mail: rosielepb@yahoo.com.br

${ }^{2}$ Universidade Nove de Julho - UNINOVE, Brasil

E-mail: luizguerrazzi@hotmail.com

${ }^{3}$ Universidade Nove de Julho - UNINOVE, Brasil

E-mail: bernardoserra@outlook.com

${ }^{4}$ Universidade Nove de Julho - UNINOVE, Brasil

E-mail: kniesscl@gmail.com
} 


\section{INTRODUÇÃO}

Nos últimos anos tem aumentado a preocupação dos pesquisadores em examinar a estrutura conceitual que compõe os estudos na disciplina de estratégia. Tentativas têm ocorrido no sentido de analisar sistematicamente um campo científico, traçar a sua evolução histórica, mapear sua estrutura intelectual, e avaliar seus pontos fortes e fracos (Nerur, Rasheed, \& Natarajan, 2008; Acedo, Barroso, \& Galan, 2006; Shafique, 2013; Pilkington, 2009), técnicas bibliométricas têm sido utilizadas para realizar estudos dessa natureza. Muitos autores têm desenvolvido estudos em gestão de negócios usando a pesquisa bibliométrica (Filardi, Barros, \& Fischmann 2014; Vieira, Machado, Brey, \& Rosseto 2012; Zhang \& Liao 2015). Ramos-Rodriguez e Ruiz Navarro (2004) realizaram um estudo para obter a estrutura intelectual da pesquisa em gestão estratégica, através da análise de citações e cocitações dos artigos publicados no Strategic Management Journal (SMJ), no período de 1980 a 2000. Neruret al. (2008) exploraram o campo da estrutura intelectual da gestão estratégica nesse mesmo periódico. Estudo semelhante foi desenvolvido por Serra, Ferreira, Almeida e Vans (2012), em que analisaram o SMJ, utilizando-se do mesmo método adotado na pesquisa de RamosRodriguez e Ruiz Navarro (2004), porém estendendo o estudo para a primeira década do século XXI.

A despeito das críticas aos trabalhos de RamosRodriguez e Ruiz Navarro (2004), Neruret al. (2008) e Serra et al. (20012) uma das principais objeções é o fato deles terem se concentrado num único periódico de estratégia, mesmo que este seja o mais conhecido e conceituado. Neste estudo, ultrapassamos esta limitação incluindo na análise um conjunto de periódicos internacionais importantes dedicados à pesquisa em estratégia: Strategic Management Journal, Strategic Organization, Journal of Economics \& Management Strategy, Long Range Planning, Strategic Entrepreneurship Journal (Thomson Reuters, 2013). Além disso, o período considerado para a análise das publicações corresponde a um período recente compreendido entre 2008 a 2013, o que nos leva a fornecer uma análise mais atual do campo da estratégia. Adotamos o método bibliométrico, sendo a amostra composta por 1213 artigos coletados nesses periódicos, como técnica de análise utilizamos a freqüência de citações, mapa de cocitações e análise fatorial para determinar os subcampos em estratégia.

Portanto, esse artigo se propõe a identificar e sistematizar as teorias que subsidiam os estudos de estratégia por meio de uma análise das publicações sobre o tema em periódicos internacionais de grande reputação na academia, pois possuem alto fator de impacto dentro da área de estratégia. A abrangência desse estudo contribuirá para ampliar o escopo do estudo de estratégia devido ao levantamento do que foi produzido e dos autores mais influentes, face às abordagens empregadas e às referências mais usadas. Essa sistematização do conhecimento vai auxiliar os pesquisadores na identificação das escolas de pensamento que predominam o campo atual da estratégia. Os pesquisadores encontrarão uma estrutura sistemática do tema estratégia, em que o conteúdo se apresenta de forma ordenada e categorizada, permitindo assim um direcionamento da pesquisa nesse campo.

$\mathrm{Na}$ primeira parte desse artigo, apresentamos a revisão teórica destacando os principais fundamentos do método bibliométrico e sua utilização no campo da administração e em especial para a área de estratégia. $\mathrm{Na}$ segunda parte detalhamos o método desse estudo e na terceira parte, revelamos os resultados. Concluímos com uma discussão detalhada, identificando as limitações do estudo, as implicações para a teoria e os caminhos para investigações futura.

\section{REFERENCIAL TEÓRICO}

O estudo bibliométrico se apresenta como uma ferramenta de análise matemática e estatística de padrões que surgem na publicação e uso de documentos (Diodato, 1994). Esse método permite mapear e gerar diferentes indicadores de tratamento e gestão da informação e do conhecimento, o que de certa forma minimiza a subjetividade inerente à indexação e recuperação das informações, produzindo conhecimento em determinada área de assunto (Guedes \& Borschiver, 2005). O método bibliométrico mostra-se apropriado à proposta desse artigo, pois nos permite identificar as principais publicações em um determinado campo ao longo do período estudado, ilustrando os principais grupos de conhecimentos e as relações gerais entre eles (Broadus, 1987; Pilkington \& Meredith, 2009).

A bibliometria utiliza-se de algumas técnicas, dentre elas destaca-se a análise de citações e de cocitações. $\mathrm{Na}$ análise de citações os autores são apresentados dentro dos temas estudados, evidenciando a predominância de temas dentro de um determinado campo. Quanto mais um documento é citado, mais influência exerce na pesquisa; uma taxa de citação em queda ao longo do tempo pode sugerir que a abordagem conceitual do documento vem perdendo relevância (Schneider \& Borlund, 2004). A análise de cocitações ocorre quando dois artigos são 
A Pesquisa em Administração Estratégica: Um Estudo Bibliométrico em Periódicos Internacionais de Estratégia no Período de 2008 a 2013

citados em conjunto em um ou mais trabalhos, medindo o grau de ligação entre esses dois artigos pelo número de documentos que os citam conjuntamente (Guedes \& Borschiver, 2005). A análise de cocitações de autores revela padrões de associação entre os autores com base em suas frequências de cocitações, o que possibilita a compreensão da evolução de uma disciplina acadêmica (White \& McCain, 1998) como também pode indicar grupos de pesquisa, que tendem a compartilhar temas teóricos e metodológicos comuns (Small \& Garfield, 1985). As citações de autores seminais proporcionam uma base para desvendar os padrões complexos de associações que existem entre eles, detectando as mudanças nas correntes intelectuais que ocorrem ao longo do tempo (Nerur et al., 2008).

A utilização da pesquisa bibliométrica nas produções científicas tem aumentado significativamente nos últimos anos, na área de administração muitos pesquisadores desenvolveram estudos utilizando-se desse método. Por exemplo, Hoffman e Holbrook (1993) realizaram um estudo de análise de cocitações de autores no Journal of Consumer Research durante seus primeiros 15 anos de publicação. Ainda em marketing os autores Chabowski, Samiee e Hult (2013), por meio de análise de citações analisaram as publicações nessa área durante um período de 30 anos. Da mesma forma Pilkington e Meredith (2009) analisaram a estrutura intelectual do campo da gestão de operações entre 1980 e 2006 . Tahai e Meyer (1999) verificaram quais os periódicos de gestão exerciam maior influência, estudo este realizado por análise de citação. Culnan, O’Reiily e Chatman (1990) observaram a estrutura intelectual da pesquisa em comportamento organizacional no período de 1972 a 1984. E, por fim, Lin e Cheng (2010) analisaram a pesquisa em aliança estratégica no período de 1999 a 2008.

Essa tendência em utilizar-se do método bibliométrico para investigar um campo de estudo é também bastante acentuado nos estudos de estratégia, a partir do estudo desenvolvido por Ramos-Rodriguez e Ruiz Navarro (2004) muitos outros surgiram, por exemplo, relacionando estratégia ao empreendedorismo (Terjesen, Hessels, \& Li, 2016), sobre melhor momento para entrada no mercado (Fosfuri, Lanzollla, \& Suarez, 2013) e, ainda, análise estratégica de modelos de negócio (Zott, \& Amit, 2013). Nos últimos anos, tem havido muitas tentativas para analisar sistematicamente o domínio do campo da gestão estratégica, traçar a sua evolução histórica, mapear sua estrutura intelectual, e avaliar seus pontos fortes e fracos (Nerur et al., 2008). Pode assim afirmar que a gestão estratégica é agora um campo estabelecido no estudo das organizações e tem testemunhado um crescimento significativo na diversidade de temas e variedade de métodos de pesquisa empregados, na medida em que o campo avançou ao longo das últimas décadas aumentou o nível de sofisticação teórica e metodológica (Hoskisson, Hitt, Wan, \& Yiuet, 1999).

\section{MÉTODO}

Esse artigo foi desenvolvido a partir de um estudo bibliométrico, ferramenta estatística que permite mapear e gerar diferentes indicadores de tratamento e gestão da informação e do conhecimento (Guedes \& Borschiver, 2005). A figura 1 representa os passos utilizados para a realização dessa pesquisa:

Figura 1. Desenho da pesquisa

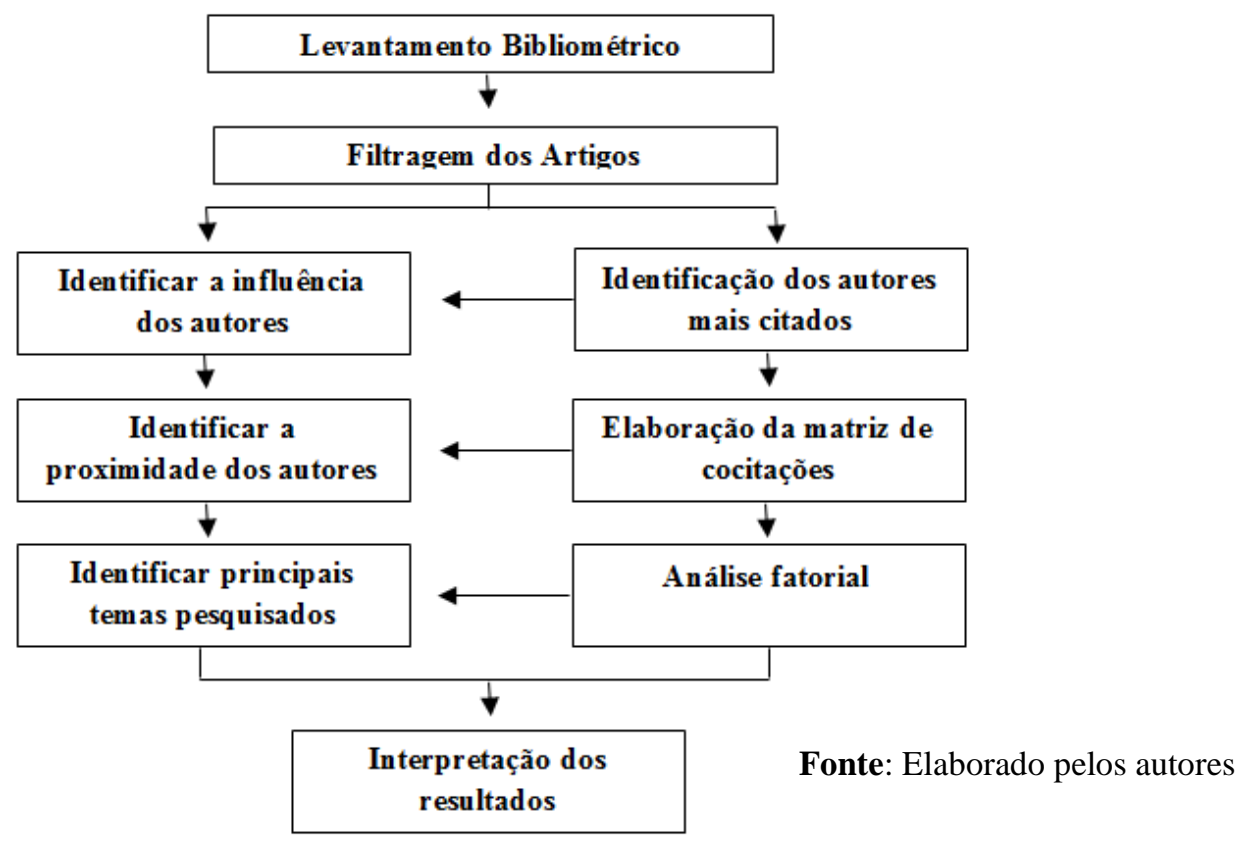


A Pesquisa em Administração Estratégica: Um Estudo Bibliométrico em Periódicos Internacionais de Estratégia no Período de 2008 a 2013

\subsection{Coleta de dados}

Por se tratar de uma pesquisa bibliométrica os dados são de fonte secundária, coletados diretamente na Web of Science (http://apps.isiknowledge.com), base de dados eletrônica pertence à ISI Web of Knowledge da Thomson Reuters, considerada como uma importante base de dados, e que possui ferramentas de busca que facilitam a pesquisa bibliométrica. Esse estudo é baseado exclusivamente em periódicos internacionais de estratégia com alto fator de impacto conforme Tabela 1. Além de referência internacional esses periódicos são específicos da área de estratégia.

Tabela 1. Lista de Periódicos

\begin{tabular}{|l|c|}
\hline \multicolumn{1}{|c|}{ Periódicos } & Fator de impacto \\
\hline Strategic Management Journal & 5,929 \\
\hline Strategic Organization & 2,472 \\
\hline Journal of Economics \& Management Strategy & 1.042 \\
\hline Long Range Planning & 4,365 \\
\hline Strategic Entrepreneurship Journal & 1,744 \\
\hline
\end{tabular}

Fonte: Thomson Reuters, 2013

\subsection{Amostra da pesquisa}

A seleção da amostra ocorreu da seguinte forma: após a escolha dos periódicos para realizar a pesquisa, foram selecionados, por meio de palavraschave, todos os artigos publicados nesses periódicos no período compreendido entre 2008 a 2013, esse procedimento resultou no levantamento de 1213 artigos. As palavras-chave usadas na coleta foram: innovation, performance, resource-based view, entrepreneurship, competitive advantage, alliances, firm performance, corporat e governance. Todos esses artigos compõem a amostra dessa pesquisa, visto que estão relacionados a estratégia. Esses artigos utilizaram cerca de 24 mil referências, sendo que para o propósito desse trabalho analisamos as referências mais citadas na amostra.

\subsection{Procedimentos de análise}

Com o objetivo de reduzirmos o número de variáveis e obtermos clusters que definem grupos de tendências ou abordagens conceituais sobre o tema, utilizamos mais de uma técnica estatística multivariada seguindo Acedo, Barroso, e Galan (2006). Na análise bibliométrica utilizamos o software Bibexcel (www.umu.se/inforsk/Bibexcel). A partir dele criamos a tabela de frequência de citação (Tabela 2) e geramos a matriz de cocitações necessária para a criação do mapa ou rede de cocitações pelo software SPSS - versão 20 (Figura 2), sendo essas análises complementadas pela análise fatorial feita também no SPSS. Para realizarmos essas análises, normalizamos a priori as referências utilizadas, visto que algumas das referências apresentavam inconsistências quanto ao nome dos autores, volumes, número, páginas e nomes dos periódicos.

Inicialmente, a análise de citações buscou identificar os principais pesquisadores e obras que contribuíram para o desenvolvimento intelectual do tema, a análise envolveu todas as referências dos 1213 artigos da amostra, sendo que foram analisados os artigos que foram citados mais de trinta e uma vez nas referências desses artigos, totalizando assim um quantitativo de 50 artigos. Em seguida, a análise de cocitações de autores nos permitiu identificarmos o grupo de artigos que influenciam os trabalhos de estratégia, bem como exibirmos suas inter-relações a partir do mapa de cocitações. Por meio dessa técnica foi possível examinar o posicionamento dos autores no campo da estratégia, isso é realizável porque a análise de cocitações permite identificar os agrupamentos a que os autores pertencem, e mostrar as principais abordagens conceituais da disciplina (White \& McCain, 1998).

Finalmente, conduzimos a análise fatorial com rotação varimax (ver Acedo, Barroso, \& Galan, 2006; Lin \& Cheng, 2010). Na pesquisa bibliométrica um fator é considerado um subcampo e representa bases teóricas a partir da análise dos autores que possuem cargas altas naquele fator (Nerur et al., 2008), artigos com proximidade conceitual ou temática terão cargas mais altas no mesmo fator. Para ser consistente com estudos anteriores (Nerur et al., 2008; Shafique, 2013) somente autores com cargas acima de 0,4 foram incluídos em um fator e representam um tema, com exceção dos trabalhos de Ocasio (1997), Stuart, Hoang e Hybels (1999), Dimaggio e Powell (1983) e Hambrick e Mason (1984), que apesar de terem cargas abaixo de 0,4 foram consideradas pertencentes ao fator dada sua força de explicação para aquele fator. A 
A Pesquisa em Administração Estratégica: Um Estudo Bibliométrico em Periódicos Internacionais de Estratégia no Período de 2008 a 2013

denominação de cada fator é feita pela identificação da vertente teórica comum aos trabalhos ali agrupados. É importante lembrar que essa análise não substitui uma leitura mais profunda dos temas caso haja interesse por parte do pesquisador em aprofundarse no estudo de um campo.

Portanto, o uso das técnicas de citação, cocitação e análise fatorial nos permitiu traçarmos a estrutura intelectual do campo de estratégia a partir de importantes periódicos internacionais da área.

\section{RESULTADOS}

Nessa seção, são apresentados os resultados da análise de citação e cocitações de autores através do levantamento das referências bibliográficas nos periódicos internacionais de estratégia durante o período de 2008 a 2013, selecionados a partir da base ISI Web Of Science. Esses periódicos produziram um total de 1213 artigos durante esses cinco anos, sendo analisados nesse trabalho aqueles que obtiveram o maior número de citações.

\subsection{Análise de citações}

A análise de citações é uma das análises de mais rápido crescimento na pesquisa bibliométrica (Sun e Grimes 2016). A Tabela 2 mostra os artigos mais citados, eles foram citados mais de trinta e uma vez nas referências dos 1213 artigos levantados nos periódicos aqui analisados. A leitura sobre esses trabalhos nos permitiu identificarmosque eles abrangem diferentes temas. Os trabalhos de Barney (1991), Nelson e Winter (1982) e Cyert e March(1963) aparecem como os mais citados, o que nos mostra a forte presença dos estudos voltados para a Visão Baseada em Recursos - RBV seja representada por trabalhos seminais como Barney (1991), ou pela extensão deste trabalho com obras que enfatizamos aspectos da aprendizagem, como é o caso de Cohen e Levinthal (1990) que ocupou a sexta posição em número de citação.

A Tabela 2 apresenta as referências dos artigos, sendo descritos os elementos centrais que o compõem, o que nos permitiu uma melhor identificação dos trabalhos analisados nessa pesquisa.

Tabela 2: Trabalhos ordenados por frequência de citação dos 1213 artigos

\begin{tabular}{|c|c|c|c|}
\hline No. & $\begin{array}{l}\text { No. De } \\
\text { citações }\end{array}$ & Documentos citados & $\begin{array}{c}\% \text { de citações } \\
\text { nos } 1213 \text { artigos }\end{array}$ \\
\hline 1 & 130 & $\begin{array}{c}\text { Barney, J. (1991) Firm resources and sustained competitive } \\
\text { advantage. Journal of Management, 17:99-120. }\end{array}$ & 10,7 \\
\hline 2 & 125 & $\begin{array}{l}\text { Nelson, R. \& Winter, S. (1982). An evolutionary theory of } \\
\text { economic change. Cambridge: Harvard University Press, } 1982 .\end{array}$ & 10,3 \\
\hline 3 & 99 & $\begin{array}{l}\text { Cyert, R. \& March, J. (1963). A behavioral theory of the firm. } \\
\text { Englewood Cliffs, NJ. }\end{array}$ & 8,2 \\
\hline 4 & 82 & $\begin{array}{c}\text { Teece, D., Pisano, G. \& Shuen, A. (1997). Dynamic capabilities } \\
\text { and strategic management. Strategic Management Journal, } \\
\text { 18:509-533. }\end{array}$ & 6,8 \\
\hline 5 & 79 & $\begin{array}{c}\text { Penrose, E. (1959). The theory of the growth of the firm. New } \\
\text { York: John Wiley. }\end{array}$ & 6,5 \\
\hline 6 & 78 & $\begin{array}{c}\text { Cohen, W. \& Levinthal, D. (1990). Absorptive capacity: A new } \\
\text { perspective on learning and innovation. Administrative Science } \\
\text { Quarterly, 35:128-152. }\end{array}$ & 6,4 \\
\hline 7 & 76 & $\begin{array}{l}\text { Porter, M. (1980). Competitive strategy: Techniques for } \\
\text { analyzing industries and competitors. New York: Free Press. }\end{array}$ & 6,3 \\
\hline 8 & 75 & $\begin{array}{l}\text { Dierickx, I. \& Cool, K. (1989). Asset stock accumulation and } \\
\text { sustainability of competitive advantage. Management Science, } \\
\text { 35:1504-1511. }\end{array}$ & 6,2 \\
\hline 9 & 74 & $\begin{array}{c}\text { March, J. (1991). Exploration and exploitation in organizational } \\
\text { learning. Organization Science, 2:71-87. }\end{array}$ & 6,1 \\
\hline 10 & 74 & $\begin{array}{l}\text { Wernerfelt, B. (1984). The Resource-Based View of the firm. } \\
\text { Strategic Management Journal, 5:171-180. }\end{array}$ & 6,1 \\
\hline 11 & 74 & $\begin{array}{c}\text { Williamson, O. (1985). The economic institutions of capitalism: } \\
\text { Firms, markets, relational contracting. New York: The Free } \\
\text { Press. }\end{array}$ & 6,1 \\
\hline 12 & 66 & $\begin{array}{l}\text { Eisenhardt, K. \& Martin, J. (2000). Dynamic capabilities: What } \\
\text { are they? Strategic Management Journal, 21:1105-1121. }\end{array}$ & 5,4 \\
\hline
\end{tabular}


A Pesquisa em Administração Estratégica: Um Estudo Bibliométrico em Periódicos Internacionais de Estratégia no Período de 2008 a 2013

\begin{tabular}{|c|c|c|c|}
\hline 13 & 64 & $\begin{array}{l}\text { Aiken, L. \& West, S. (1991).Multiple regression: Testing and } \\
\text { interpreting interactions. Newbury Park, CA: Sage. }\end{array}$ & 5,3 \\
\hline 14 & 62 & $\begin{array}{c}\text { Kogut, B. \& Zander, (1992). U. Knowledge of the firm, } \\
\text { combinative capabilities, and the replication of technology. } \\
\text { Organization Science, 3:383-397. }\end{array}$ & 5,1 \\
\hline 15 & 60 & $\begin{array}{c}\text { Teece, D. (1986) Profiting from technological innovation: } \\
\text { Implications for integration, collaboration, licensing and public } \\
\text { policy.Research Policy, 15: 285-305. }\end{array}$ & 4,9 \\
\hline 16 & 56 & $\begin{array}{c}\text { Levinthal, D. \& March, J. (1993). The myopia of learning. } \\
\text { Strategic Management Journal, 14: 95-112. }\end{array}$ & 4,6 \\
\hline 17 & 56 & $\begin{array}{l}\text { Thompson, J. (1967). Organizations in action: Social science } \\
\text { bases of administrative theory. New York: McGraw-Hill. }\end{array}$ & 4,6 \\
\hline 18 & 55 & $\begin{array}{l}\text { Dimaggio, P. \& Powell, W. (1983). The iron cage revisited: } \\
\text { Institutional isomorphism and collective rationality in } \\
\text { organizational fields. American Sociological Review, 48:147- } \\
160 .\end{array}$ & 4,5 \\
\hline 19 & 50 & $\begin{array}{l}\text { Barney, J. (1986). Strategic Factor Markets: Expectations, Luck, } \\
\text { and Business Strategy. Management Science, 32:1231-1241. }\end{array}$ & 4,1 \\
\hline 20 & 50 & $\begin{array}{c}\text { Jensen, M. \& Meckling, W. (1976) Theory of the firm: } \\
\text { Managerial behavior, agency costs and ownership structure. } \\
\text { Journal of Financial Economics, 3: 305-360. }\end{array}$ & 4,1 \\
\hline 21 & 49 & $\begin{array}{l}\text { Eisenhardt, K. M. (1989). Building theories from case study } \\
\text { research. Academy of Management Review, 14:532-550. }\end{array}$ & 4,0 \\
\hline 22 & 49 & $\begin{array}{l}\text { March, James G. \& Simon, H. (1958). Organizations. Wiley: } \\
\text { New York, 1958. }\end{array}$ & 4,0 \\
\hline 23 & 49 & $\begin{array}{c}\text { Pfeffer, J. \& Salancik, G. (1978). The external control of } \\
\text { organizations: A resource dependence perspective. New York: } \\
\text { Harper and Row. }\end{array}$ & 4,0 \\
\hline 24 & 48 & $\begin{array}{l}\text { Grant, M. (1996). Toward a Knowledge-Based Theory of the } \\
\text { Firm. Strategic Management Journal, 17:109-122. }\end{array}$ & 4,0 \\
\hline 25 & 48 & $\begin{array}{c}\text { Peteraf, M. (1993). The Cornerstones of Competitive } \\
\text { Advantage: A Resource-Based View. Strategic Management } \\
\text { Journal, 14:179-91. }\end{array}$ & 4,0 \\
\hline 26 & 47 & $\begin{array}{c}\text { Williamson, O. (1975). Markets and hierarchies, New York: } \\
\text { Free Press. }\end{array}$ & 3,9 \\
\hline 27 & 46 & $\begin{array}{c}\text { Hambrick, D. \& Mason, P. (1984). Upper echelons: the } \\
\text { organization as a reflection of its top managers. Academy of } \\
\text { Management Review, 9: 193-206. }\end{array}$ & 3,8 \\
\hline 28 & 45 & $\begin{array}{l}\text { Dyer, J. \& Singh, H. (1998). The relational view: Cooperative } \\
\text { strategy and sources of interorganizational competitive } \\
\text { advantage. Academy of Management Review, 23: 660-679. }\end{array}$ & 3,7 \\
\hline 29 & 44 & $\begin{array}{l}\text { Stinchcombe, L. (1965). Social Structure and Organizations. In: } \\
\text { J. G. March (Ed.), Handbook, of Organizations (pp: 142-193). } \\
\text { Chicago, IL: Rand McNally \& Company. }\end{array}$ & 3,6 \\
\hline 30 & 43 & $\begin{array}{c}\text { Helfat, E. \& Peteraf, A. (2003). The Dynamic Resource-Based } \\
\text { View: Capability Lifecycles. Strategic Management Journal, 24: } \\
\text { 997-1010. }\end{array}$ & 3,5 \\
\hline 31 & 43 & $\begin{array}{c}\text { Levitt B. \& March J. (1988). Organizational learning. Annual } \\
\text { Review of Sociology, 14:319-340. }\end{array}$ & 3,5 \\
\hline 32 & 43 & $\begin{array}{c}\text { Tushman M. \& Anderson, P. (1986). Technological } \\
\text { discontinuities and organizational environments. Administrative } \\
\text { Science Quarterly, 31: 439-465. }\end{array}$ & 3,5 \\
\hline 33 & 42 & $\begin{array}{c}\text { Amit, R. \& Shoemaker, P. (1993). Strategic assets and } \\
\text { organizational rent. Strategic Management Journal, 14: 33-46. }\end{array}$ & 3,5 \\
\hline 34 & 41 & $\begin{array}{l}\text { Henderson, R. \& Clark, K. (1990). Architectural Innovation: The } \\
\text { reconfiguration of existing product technologies and the failure of } \\
\text { establishment firms. Administrative Science Quarterly, 35: 9-30. }\end{array}$ & 3,4 \\
\hline
\end{tabular}


A Pesquisa em Administração Estratégica: Um Estudo Bibliométrico em Periódicos Internacionais de Estratégia no Período de 2008 a 2013

\begin{tabular}{|c|c|c|c|}
\hline 35 & 40 & $\begin{array}{c}\text { Ocasio, W. (1997). Towards an attention-based view of the firm. } \\
\text { Strategic Management Journal, 18:187-206. }\end{array}$ & 3,3 \\
\hline 36 & 39 & $\begin{array}{l}\text { Burt, R. (1992). Structural holes: The social structure of } \\
\text { competition. Cambridge, MA: Harvard University Press. }\end{array}$ & 3,2 \\
\hline 37 & 37 & $\begin{array}{c}\text { Szulanski, G. (1996). Exploring internal stickiness: Impediments } \\
\text { to the transfer of best practice within the firm. Strategic } \\
\text { Management Journal, 17: 27-43. }\end{array}$ & 3,0 \\
\hline 38 & 37 & $\begin{array}{l}\text { Zollo, M. \& Winter, G. (2002). Deliberate learning and the } \\
\text { evolution of dynamic capabilities. Organization Science, } \\
\text { 13:339-351. }\end{array}$ & 3,0 \\
\hline 39 & 36 & $\begin{array}{c}\text { Leonardbarton, D. (1992). Core Capabilities and Core Rigidities } \\
\text { - A Paradox In Managing New Product Development. Strategic } \\
\text { Management Journal, 13:111-125. }\end{array}$ & 3,0 \\
\hline 40 & 35 & $\begin{array}{l}\text { Porter M. (1985). Competitive Advantage. Free Press: New } \\
\text { York. }\end{array}$ & 2,9 \\
\hline 41 & 34 & $\begin{array}{l}\text { Eisenhardt, M. (1989). Making Fast Strategic Decisions in High- } \\
\text { Velocity Environments. Academy of Management Journal, 32: } \\
\text { 543-576. }\end{array}$ & 2,8 \\
\hline 42 & 34 & $\begin{array}{c}\text { Powell, W. Koput, K. \& Smith-Doerr, L. (1996). } \\
\text { Interorganizational collaboration and the locus of innovation: } \\
\text { Networks of learning in biotechnology. Administrative Science } \\
\text { Quartery, 41:116-145. }\end{array}$ & 2,8 \\
\hline 43 & 34 & $\begin{array}{l}\text { Sirmon, G., Hitt, A. \& Ireland, D. (2007). Managing firm } \\
\text { resources in dynamic environments to create value: Looking } \\
\text { inside the black box. Academy of Management Review, 32: 273- } \\
292 .\end{array}$ & 2,8 \\
\hline 44 & 34 & $\begin{array}{l}\text { Stuart, E., Hoang, H. \& Hybels, C. (1999). Interorganizational } \\
\text { endorsements and the performance of entrepreneurial ventures. }\end{array}$ & 2,8 \\
\hline 45 & 34 & $\begin{array}{l}\text { Teece, J. (2007). Explicating dynamic capabilities: The nature } \\
\text { and microfoundations of (sustainable) enterprise performance. } \\
\text { Strategic Management Journal, 28:1319-1350. }\end{array}$ & 2,8 \\
\hline 46 & 33 & $\begin{array}{c}\text { Greene, H. (2003). Econometric Analysis, Saddle River, NJ: } \\
\text { Prentice Hall. }\end{array}$ & 2,7 \\
\hline 47 & 32 & $\begin{array}{c}\text { Hannan, M. \& Freeman, J. (1984). Structural inertia and } \\
\text { organizational change. American Sociological Review, 49: 149- } \\
64 .\end{array}$ & 2,6 \\
\hline 48 & 32 & $\begin{array}{c}\text { Helfat, E., Finkelstein, S., Mitchell, W., Peteraf, A., Singh, H., } \\
\text { Teece, D. J. \& Winter, G. (2007). Dynamic capabilities. } \\
\text { Understanding Strategic Change in Organizations. Oxford: } \\
\text { Blackwell Publishing. }\end{array}$ & 2,6 \\
\hline 49 & 32 & $\begin{array}{l}\text { Lieberman, B. \& Montgomery, B. (1988). 1St-mover } \\
\text { advantages. Strategic Management Journal, 9:41-58. }\end{array}$ & 2,6 \\
\hline $\mathbf{5 0}$ & 31 & $\begin{array}{l}\text { Katila, R. \& Ahuja, G. (1992). Something old, something new: } \\
\text { A longitudinal study of search behavior and new product } \\
\text { introduction. Academy of Management Journal, 45: 1183-1194. }\end{array}$ & 2,5 \\
\hline
\end{tabular}

Fonte: Dados coletados do ISI Web of Knowledge. Elaborado pelos autores

Se tomarmos como ponto de análise a comparação entre essa pesquisa e a pesquisa realizada por Ramos-Rodriguez e Ruiz Navarro (2004) é possível observarmos que alguns dos autores que receberam um número significativo de citações no SMJ no período de 1980 a 2000, quando analisados sob a luz dos principais periódicos internacionais de estratégia,esses trabalhos não aparecem dentro dos mais citados. O exemplo disso são os trabalhos de Rumelt (1974), Porter (1985) e Chandler (1985), que ocupavam uma posição de destaque em número de citações em Ramos-Rodriguez e Ruiz Navarro (2004), ocupando segundo, terceiro e quarto lugar respectivamente no ranking, porém em nossa pesquisa 
A Pesquisa em Administração Estratégica: Um Estudo Bibliométrico em Periódicos Internacionais de Estratégia no Período de 2008 a 2013

eles perderam essa posição. Por outro lado, alguns autores como Teece, Pisano e Shuen (1997), Cohen e Levinthal (1990), March (1991), emergiram nessa pesquisa com um número significativo de citações, enquanto que em Rodriguez e Ruiz Navarro (2004) eles não haviam sido enquadrados entre os mais citados. Isto mostra que no trabalho de RamosRodriguez e Ruiz Navarro (2004) há uma maior ênfase em referências clássicas provenientes das escolas do design, planejamento e posicionamento, enquanto que no nosso estudo, temas mais contemporâneos como aprendizagem organizacional e capacidades dinâmicas aparecem em destaque com maior número de vezes citados.

\subsection{Análise de cocitações}

O diagrama obtido pelo escalonamento multidimensional (MDS), baseado na frequência de cocitações, elaborado a partir do uso do SPSS e apresentado na Figura 2, representa a proximidade existente entre os autores, ou seja, quanto mais próximo os autores estiverem uns dos outros significa que foram mais frequentemente citados juntamente em outros trabalhos. Portanto, a proximidade entre os círculos representa a co-participação entre trabalhos, quanto menor a distância mais frequentemente os artigos são cocitados (Shafique 2013). O diâmetro do círculo é proporcional à frequência de citação de cada trabalho, quando o círculo é maior significa que o número de citações é maior.

Para uma melhor visualização dos trabalhos, a escala multidimensional (MDS) foi gerada a partir das 35 referências mais citadas (Figura 2), tendo sido citadas mais de quarenta vezes no total das referências analisadas. Essa forma de apresentação nos permitiu uma melhor compreensão das relações existentes entre os trabalhos mais citados, pois evidenciou a estrutura das ligações intelectuais entre esses trabalhos, por meio da proximidade entre eles no diagrama. A discussão dos resultados acerca dos clusters identificados no mapa de cocitações será apresentada na seção seguinte.

Figura 2. Diagrama MDS - Mapa de cocitações de autores

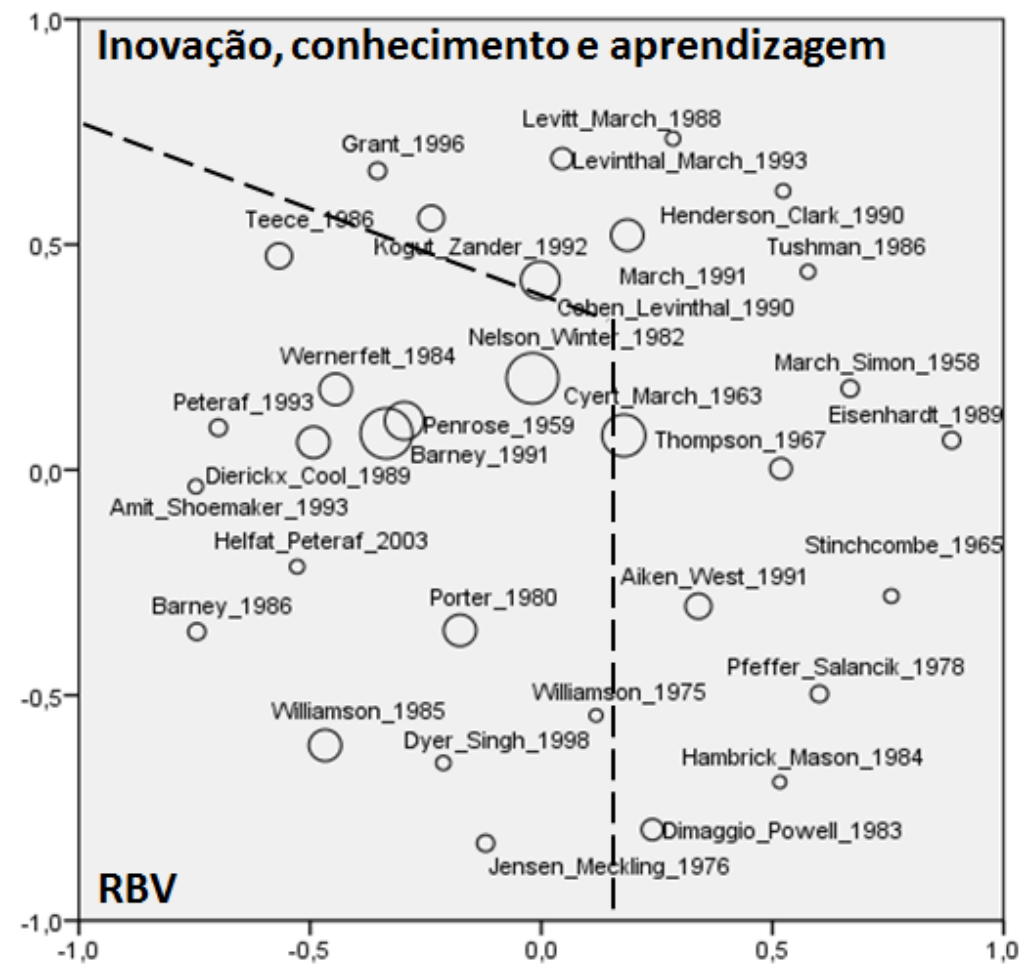

Fonte: Processado pelo SPSS (versão 20). Elaborado pelos autores.

\subsection{Análise fatorial}

A análise fatorial pode ser utilizada para determinar subcampos ou temas (Lin \& Cheng, 2010), a carga fatorial é um indicador do grau no qual um artigo pertence ao fator e o fator representa o sub- campo ou tema (Nerur et al., 2008). Artigos que são conceitualmente próximos tendem a ter cargas mais altas no mesmo fator. Nesse estudo a análise fatorial foi conduzida sobre os 50 trabalhos mais citados, sendo identificados três fatores (Tabela 3) com uma variância explicada de 53,3\%, esses fatores refletem 
A Pesquisa em Administração Estratégica: Um Estudo Bibliométrico em Periódicos Internacionais de Estratégia no Período de 2008 a 2013

os principais temas pesquisados dentro do campo da estratégia. A interpretação é semelhante aos clusters anteriores gerados pela matriz de cocitações e a discussão desses clusters também será apresentada na seção seguinte. Apesar de cada obra ter sido atribuída a um único fator, há uma proximidade entre esses clusters, ou seja, determinados autores possuem cargas altas em mais de um fator, é razoável afirmar que isso ocorre porque algumas obras são conceitualmente próximas de outras mesmo que nessa pesquisa estejam representadas em clusters diferentes.

Tabela 3. Sumário da Análise de Fatores

\begin{tabular}{|c|c|c|c|c|c|}
\hline \multicolumn{6}{|c|}{ CLUSTER } \\
\hline $\begin{array}{c}\text { Resource Based View - } \\
\text { RBV }\end{array}$ & & $\begin{array}{c}\text { Inovação, Conhecimento, } \\
\text { Aprendizagem }\end{array}$ & & Capability & \\
\hline Amit \&Shoemaker(1993) & 0,882 & Levitt \& March (1988) & 0,814 & Teece (2007) & 0,605 \\
\hline Peteraf (1993) & 0,863 & Hannan \& Freeman (1984) & 0,767 & Teece et al. (1997) & 0,582 \\
\hline Sirmon et al. (2007) & 0,819 & March (1991) & 0,747 & $\begin{array}{l}\text { Eisenhardt \& Martin } \\
(2000)\end{array}$ & 0,427 \\
\hline Wernerfelt (1984) & 0,806 & Levinthal \& March (1993) & 0,742 & Ocasio (1997) & 0,288 \\
\hline Dierickx \& Cool (1989) & 0,804 & March \& Simon (1958) & 0,710 & & \\
\hline Penrose (1959) & 0,754 & Kogut \&Zander (1992) & 0,672 & & \\
\hline Porter (1980) & 0,751 & Cohen \& Levinthal (1990) & 0,666 & & \\
\hline Porter (1985) & 0,724 & Szulanski (1996) & 0,647 & & \\
\hline $\begin{array}{c}\text { Lieberman \& } \\
\text { Montgomery (1988) }\end{array}$ & 0,694 & Grant (1996) & 0,646 & & \\
\hline Helfat \& Peteraf (2003) & 0,690 & Powell et al. (1996) & 0,636 & & \\
\hline Helfat et al. (2007) & 0,623 & Cyert \& March(1963) & 0,620 & & \\
\hline Barney (1991) & 0,607 & Thompson (1967) & 0,599 & & \\
\hline Teece (1986) & 0,556 & Eisenhardt(1989) & 0,599 & & \\
\hline Williamson (1975) & 0,538 & Tushman (1986) & 0,540 & & \\
\hline Nelson \& Winter (1982) & 0,468 & Eisenhardt(1989a) & 0,524 & & \\
\hline $\begin{array}{c}\text { Jensen \& Meckling } \\
(1976)\end{array}$ & 0,420 & Henderson \& Clark (1990) & 0,517 & & \\
\hline & & Salancik \& Pfefer(1978) & 0,511 & & \\
\hline & & LeonardBarton (1992) & 0,508 & & \\
\hline & & Stinchcombe (1965) & 0,507 & & \\
\hline & & Aiken \& West (1991) & 0,507 & & \\
\hline & & Greene (2003) & 0,500 & & \\
\hline & & Burt (1992) & 0,479 & & \\
\hline & & Dyer \& Singh (1998) & 0,474 & & \\
\hline & & Williamson (1985) & 0,407 & & \\
\hline & & Stuart et al. (1999) & 0,389 & & \\
\hline & & Dimaggio \& Powell (1983) & 0,361 & & \\
\hline & & Hambrick \& Mason (1984) & 0,349 & & \\
\hline Variância explicada & $22,9 \%$ & & $22,7 \%$ & & $7,7 \%$ \\
\hline
\end{tabular}

Fonte: Elaborado pelos autores

\section{DISCUSSÃO}

O objetivo dessa pesquisa foi entender a teia que forma o campo científico da estratégia por meio de uma leitura sobre as principais produções científicas publicadas nos cinco importantes periódicos internacionais de estratégia, com base no pressuposto de que a estrutura intelectual de um campo pode ser medida empiricamente pela bibliometria. Portanto a natureza desse trabalho foi viabilizada através do método bibliométrico, que nos permitiu pela análise de citação, cocitaçõese a análise fatorial identificarmos as principais tendências teóricas desenvolvidas dentro do campo da estratégia, bem como a conexão existente entre os principais autores.

As análises realizadas nessa pesquisa nos forneceram evidências que sustentam a ideia da 
A Pesquisa em Administração Estratégica: Um Estudo Bibliométrico em Periódicos Internacionais de Estratégia no Período de 2008 a 2013

abrangência de várias frentes de investigação no campo da estratégia, representado nessa pesquisa pelos clusters: Resource Based View - RBV, Inovação, Conhecimento e Aprendizagem e Capability. Embora adotamos nessa pesquisa a classificação das obras dos autores mais influentes no campo da estratégia dentro desses clusters, há uma proximidade desses autores entre si, ou seja, as obras que tratam de inovação, conhecimento e aprendizagem são uma extensão dos estudos de RBV tendo uma ligação forte com os autores que tratam da RBV.

Os trabalhos de Eisenhardt (1989) e Aiken (1991) apesar de terem sido citados não representam um grupo por tratar de trabalhos voltados para métodos de pesquisa. O trabalho de Porter (1980) e Porter (1985) embora citados oferecem apenas uma referência de contraponto em estratégia competitiva.

\subsection{A visão baseada em recursos - RBV}

A RBV aparece nessa pesquisa representada pelas obras consideradas seminais, tendo como mais citados os trabalhos de Barney (1991), Nelson e Winter (1982), Penrose (1959), Dierickx e Cool (1989), Wernefelt (1984), seguido dos trabalhos (Teece, 1986; Barney, 1986; Peteraf, 1993; Sirmon, 2007; Helfat\& Peteraf, 2003; Amit \& Shoemaker, 1993). O artigo de Barney (1991) afirma que os recursos estratégicos das empresas, incluem todos os ativos, capacidades, processos organizacionais, atributos, informação e conhecimento, que habilitem a empresa a implementar estratégias que melhorem sua eficiência e efetividade. Esse artigo aparece como o mais citado dentre os artigos analisados nessa pesquisa o que representa ainda um crescimento da influência desse autor para o campo científico da estratégia, pois se tomarmos o trabalho de Ramos-Rodriguez e Ruiz Navarro (2004) como parâmetro, o artigo de Barney apesar de bastante citado, ocupava apenas a décima terceira posição em número de citação naquele trabalho.

Os artigos seminais da RBV exerceram um papel importante na origem de outras tendências do campo de estratégia. A obra de Nelson e Winter (1982) aparece como um elo entre as obras clássicas da RBV e as obras relacionadas ao conhecimento/aprendizagem/inovação. Essa configuração também foi constatada por Acedo, Barroso e Galan (2006) quando analisou as principais tendências da RBV. Penrose (1959) realiza uma análise teórica do crescimento da empresa destacando que o tamanho é apenas um subproduto desse crescimento, a ênfase é dada aos recursos internos da empresa em que o crescimento das empresas consiste num processo dinâmico de interação da gestão com esses recursos. Wernerfelt (1984) enfatiza que o estudo da estratégia deve analisar a posição dos recursos nas organizações e olhar para as opções estratégicas que nascem dessa análise, o que de certa forma implica numa nova maneira de gerenciar os recursos. Para Dierickx e Cool (1989) a dimensão essencial da formulação de estratégia pode ser identificada como a tarefa de fazer escolhas adequadas sobre os gastos estratégicos visando acumular recursos e habilidades necessárias.

Portanto, os trabalhos representados nesse cluster reforçam a lógica do quanto ainda são importantes os estudos no campo da estratégia que analisam os recursos como forças para que as empresas possam obter vantagem competitiva.

\subsection{Inovação, conhecimento e aprendizagem na pesquisa em estratégia}

Os trabalhos que aparecem nesse grupo refletem a visão baseada no conhecimento, aprendizagem organizacional e inovação, podendo ser considerados como uma extensão dos trabalhos da RBV (Acedo et al., 2006). Essa abordagem é representada nessa pesquisa pelas obras de Cyert e March (1963), Cohen e Levinthal (1990), March (1991), Kogut e Zander (1992) e Levinthal e March (1993), Tompson (1967), Grant (1996), Stinchombe (1965) que estão entre os trabalhos mais citados, além de outros (Burt, 1992; Greene, 2003, Hannan \& Freeman, 1984; Henderson \& Clark, 1990; Leonardbarton, 1992; Levitt \& March, 1988, Powell, 1996; Stuart, 1999; Szulanski, 1996; Tushman, 1986).

A obra de Cyert e March (1963) foi bastante representativa nesse grupo, ela ocupa a terceira posição em número de citações dentre os artigos aqui analisados. Essa obra fornece observações detalhadas das maneiras pelas quais as organizações tomam decisões, pela análisenos procedimentos que envolvem esse processo. Ela defende que uma maneira de compreender a tomada de decisão organizacional é completar o estudo microeconômico de fatores estratégicos de mercado com um exame do funcionamento interno da empresa, para estudar os efeitos da estrutura organizacional e práticas convencionais sobre desenvolvimento de metas organizacionais, expectativas organizacionais, escolhas e controle organizacional.

O conceito apresentando na obra de Cohen e Levinthal (1990) está relacionado a capacidade de absorção (absorptive capacity) conceito esse que defende a lógica da aprendizagem como fundamental para que as empresas possam se adaptar as mudanças do ambiente externo. Uma revisão da literatura de absorptive capacity revelou que a natureza intrínseca do conhecimento organizacional, o papel dos indivíduos e suas interações sociais não foram suficientemente problematizados, e questões sobre como a absorptive capacity surge como uma capacidade organizacional ficaram sem respostas, os antecedentes organizacionais da absorptive capacity 
A Pesquisa em Administração Estratégica: Um Estudo Bibliométrico em Periódicos Internacionais de Estratégia no Período de 2008 a 2013

agem como um conjunto de oportunidades para as pessoas aprenderem e inovarem e tem importância em relação aos seus comportamentos de aprendizagem (Martinkenaite \& Breunig, 2016).

O trabalho de March (1991) também exerce uma forte influência nesse grupo e consiste nos conceito de exploration e exploitation. Exploration significa que a empresa deve potencializar o conhecimento existente para garantir estabilidade e desempenho, enquanto que exploitation a empresa deve gerar novos conhecimentos criando condições para a inovação e adaptação as mudanças necessárias para garantir o futuro dos negócios. O conhecimento dentro de uma organização é formado por informações e know-how, mantido não somente pelos indivíduos, mas também pela cooperação entre eles, como novas formas de cooperação não podem ser facilmente adquiridas, o crescimento ocorre através da construção de relações sociais que existem atualmente em uma empresa, as empresas criam novos conhecimentos, a partir dos laços criados entre os membros da organização (Kogut \& Zander, 1992). No processo de aprendizagem as organizações devem compatibilizar o desenvolvimento de novos conhecimentos e explorar competências atuais, as organizações enfrentam esse dilema através da simplificação e especialização o que contribui para a miopia na aprendizagem (Levinthal \& March, 1993).

A partir da análise dos trabalhos evidenciados nesse grupo podemos afirmar a crescente influência das teorias da RBV em que a pesquisa em estratégia é fortemente orientada para os aspetos internos da empresa como fonte de vantagem competitiva, em especial pela aprendizagem, conhecimento e inovação. As obras inseridas nesse grupo defendem a lógica que o conhecimento é o mais importante recurso estratégico da organização. Especificamente, a literatura tem muitas vezes utilizado da teoria da aprendizagem organizacional no subcampo da formulação da estratégia corporativa em estudos como, por exemplo, de gestão estratégica internacional (White, Guldiken, Hemphill, Wu, \& Khoobdeh, 2016).

\subsection{Capability na pesquisa em estratégia}

Enquanto a RBV e aprendizagem organizacional constituíram os estudos de capacidade dinâmica nos estágios iniciais de sua evolução, a literatura mais recente indica que o campo está se deslocando em direção a uma agenda mais integrada de pesquisa, envolvendo também o campo da teoria da organização (Vogel \& Guttel, 2013). Nessapesquisaa capacidade dinâmica é representado pelos trabalhos de Teece et al. (1997), Teece (2007), Eisenhardt e Martin (2000), Zollo e Winter (2002), conforme apresentado na análise fatorial (Tabela 3). A RBV buscou explicar como as empresas alcançam a vantagem competitiva e como ela se torna sustentável ao longo dos anos, porém essa teoria não explicou adequadamente como obter essa vantagem competitiva em ambientes de rápidas mudanças. Teece et al. (1997) desenvolveu o conceito de capacidades dinâmicas, que consiste na análise das fontes e os métodos de criação e captura de riqueza por empresas privadas que operam em ambientes de rápida mudança tecnológica, essa criação decorre do aperfeiçoamento de processos tecnológicos organizacionais e gerenciais dentro da empresa. Em Teece (2007) o autor buscou especificar a natureza e microfundamentos das capacidades necessárias para sustentar o desempenho superior das empresas em um ambiente de inovações rápidas, para ele a capacidade dinâmica permite que as empresas criem, implantem e protejam os ativos intangíveis que suportem a vantagem competitiva do negócio em longo prazo. Os microfundamentos das capacidades dinâmicas são difíceis de serem desenvolvidos e implantados. Empresas com fortes capacidades dinâmicas são intensamente empreendedora, são adaptativas e se utilizam da inovação e colaboração com outras empresas e instituições. A gestão da inovação por si só é insuficiente para gerar o sucesso sem as capacidades dinâmicas de uma empresa para propositadamente criar, ampliar ou modificar a sua base de recursos (Lin, Su \& Higgins, 2016).

Eisenhardt e Martin (2000) definem capacidade dinâmica como sendo as rotinas organizacionais e estratégicas pelas quais as empresas conquistam novos recursos como os mercados emergem, colidem, evoluem e morrem. As capacidades dinâmicas alteram a base de recursos da empresa, o que inclui seus ativos organizacionais físicos e humanos, essa capacidade de alterar recursos representa um valor para a vantagem competitiva. É a capacidade de uma organização propositadamente criar e estender ou modificar seus recursos base (Helfat, Finkelstein, Mitchell, Peteraf, Singh, Teece, Winter \& 2007; Helfat \& Peteraf, 2009). Zollo e Winter (2002) foca na aprendizagem organizacional como a fonte de capacidade dinâmica, esses mecanismos de aprendizagem são a acumulação de experiência, a articulação e codificação do conhecimento. A codificação do conhecimento para esses autores se torna mecanismo superior com relação ao acúmulo de expetise como a freqüência e a homogeneidade das tarefas são reduzidas.

Portanto, as teorias no campo da estratégia tem variado ao longo dos anos, White et al. (2016) ao analisarem a tendência da pesquisa em gestão estratégica internacional mostraram que a visão baseada no conhecimento, a RBV, aprendizagem organizacional foram aplicadas de forma consistente no período de 2000 a 2013. O que de certa forma se confirma a aplicação dessas teorias ao campo da estratégia. 
A Pesquisa em Administração Estratégica: Um Estudo Bibliométrico em Periódicos Internacionais de Estratégia no Período de 2008 a 2013

\section{CONSIDERAÇÕES FINAIS}

Este estudo teve como objetivo investigar as abordagens teóricas que têm fundamentado o estudo de estratégia nos últimos anos. Apesar de a literatura apontar trabalhos que tenham estudado a evolução do campo de estratégia através do método bibliométrico (Ramos-Rodriguez \& Ruiz-Navarro, 2004; Serra et al., 2012; Nerur et al., 2008) o que se observa é que nesses trabalhos foram avaliados somente os artigos publicados no Strategic Management Journal. Essa pesquisa se mostrou relevante e abrangente por ter analisado os artigos publicados em cinco importantes periódicos internacionais de estratégia. Além disso, traz uma perspectiva atual do campo, com assuntos mais contemporâneos e ligações com temáticas do campo de estudos organizacionais, haja vista que o período analisado é recente, compreendido entre 2008 a 2013.

Essa pesquisa nos permitiu identificarmos as abordagens teóricas predominantes nos estudos de estratégia: a Resource Based View - RBV, Inovação, Conhecimento, Aprendizagem e Capability. Os resultados confirmam a tendência apontada por Ramos-Rodriguez e Ruiz-Navarro (2004) da emergência da RBV, pois foi identificada a predominância também dessa teoria nos trabalhosaqui analisados. Vinte anos mais tarde, a RBV é amplamente reconhecida como uma das teorias mais importantes e poderosas para descrever, explicar e predizer relações organizacionais (Barney, Ketchen, \& Wright, 2011).

Essa pesquisa também confirma a relação de estudos de RBV com conhecimento e aprendizagem organizacional, e com inovação, apontado no estudo de Serra et al. (2012). A integração da RBV com outras perspectivas pode lançar uma nova luz sobre questões fundamentais dentro da RBV e o campo da gestão estratégica em geral (Barney et al., 2011).

A Teoria da Agência (Jensen \& Meckling, 1976) e a Teoria Institucional (DiMaggio \& Powell, 1983), também apareceram nessa pesquisa. A Teoria da Agência mostra a importância e atualidade dos problemas da relação entre o principal agente e os gerentes. A presença do trabalho de DiMaggio e Powell (1983) mostra uma integração da estratégia com a teoria das organizações, bem como, o fato da estratégia ser impactada e influenciada pelas forças institucionais. Essa é uma descoberta nova nessa pesquisa em relação a Ramos-Rodriguez e RuizNavarro (2004) o que mostra a evolução do campo da estratégia convergindo com o campo da Teoria das Organizações. Essa integração havia sido elucidada por Vasconcelos e Cyrino (2000), os autores apontaram que as contribuições teóricas e conceituais tanto no campo da administração estratégica quanto em teoria das organizações são responsáveis por essa convergência. Em estratégia os autores destacam os estudos que buscam identificar os fatores de sucesso ou fracasso das empresas em diferentes contextos. No campo da teoria organizacional os autores destacam os estudos nas ciências sociais sobre a natureza dinâmica dos processos de mudança organizacional. Vasconcelos e Cyrino (2000) defendem que apesar das diferenças teóricas metodológicas entre essas duas correntes a integração se justifica para a disciplina administração estratégica que visa informar e orientar a prática a rejeição dos principais modelos de planejamento estratégico fez soar um alarme, apontando a necessidade da redefinição do seu escopo e de sua inter-relação com outras disciplinas, levando em última instância a uma aproximação entre a fundamentação econômica e a descrição sociológica. (Vasconcelos \& Cyrino, 2000).

Nag, Hambrick e Chen (2007) argumentaram que no campo da estratégia o consenso ainda é frágil e que apesar da forte influência da economia, seus estudos se sobrepõem aos de outros campos. A estratégia, como apontado por esses autores, atua como aglutinadora intelectual de diversas possibilidades de abordagens integradas, o que de certa forma é também apresentado neste estudo bibliométrico. Portanto esse estudo contribui com o melhor entendimento das escolas de pensamento que atualmente permeiam o campo da estratégia, para que assim possam orientar futuros estudos nesse campo com base nas lacunas conceituais identificadas.

A limitação desse trabalho se relaciona as características do método bibliométrico. Primeiro em relação aos periódicos selecionados, visto que eles abrangem somente uma parte dos estudos de estratégia que têm sido publicados, porém a amostra dessa pesquisa não pretende ser exaustiva, mas representativa, estudos futuros podem se utilizar de outros periódicos não selecionados nessa pesquisa. Outra limitação se deve a análise de citações, de certa forma a decisão de analisarapenas as obras mais citadas, apesar de serem expressivas, ignora o que se passa no campo científico a partir do olhar nasdemais obras que não se configuram como as mais citadas. Essaconstatação leva a proposição de futuras pesquisas em que possa se voltar o estudo também para as obras que não se enquadram entre as mais citadas. Um aspecto a ser considerado é que, nessa pesquisa não foram analisados o conteúdo dos artigos levantados, sugerimos que pesquisas futuras possam aprofundar nos temas dos artigos, estabelecendo assim uma melhor compreensão dos mesmos. 
A Pesquisa em Administração Estratégica: Um Estudo Bibliométrico em Periódicos Internacionais de Estratégia no Período de 2008 a 2013

\section{REFERÊNCIAS}

Acedo, F., Barroso, C. \& Galan, J. (2006).The resource-based theory: Dissemination and main trends. Strategic Management Journal, 27, 621636.

Amit, R. \& Shoemaker, P. (1993) Strategic assets and organizational rent.Strategic Management Journal, 14, 33-46.

Ahuja, G. (2000). Collaboration networks, structural holes, and innovation: A longitudinal study. Administrative Science Quarterly, 45, 425-455.

Aiken, L. \& West, S. (1991). Multiple regression: Testing and interpreting interactions. Newbury Park, CA: Sage.

Barney, J. (1986). Strategic Factor Markets: Expectations, Luck, and Business Strategy. Management Science, 32, 1231-1241.

Barney, J., Ketchen, D. \& Wright, M. (2011). The future of resource-based theory: Revitalization or decline? Journal of Management, 37, 1299-1315.

Barney, J. (1991). Firm resources and sustained competitive advantage. Journal of Management, 17, 99-120.

Broadus, R. (1987).Toward definition of bibliometrics. Scientometrics, 12, 73-79.

CAPES. 2010. Sistema WebQualis. Disponível em: http://qualis.capes.gov.br/webqualis/publico/doc umentosDeArea.seam?conversationPropagation= begin Acesso em 01 de Junho de 2013.

Burt, R. (1992).Structural holes: The social structure of competition. Cambridge, MA: Harvard University Press.

Chabowski, B., Samiee, S., \& Hult, G. (2013). A bibliometric analysis of the global branding literature and a research agenda. Journal of International Business Studies, 44(6), 622-634.

Cohen, W. \& Levinthal, D. (1990) Absorptive capacity: A new perspective on learning and innovation. Administrative Science Quarterly, $35,128-152$.

Culnan, J.,O’ReiilyC. \& Chatman, J. (1990). Intellectual structure of research in organizational behavior, 1972-1984: A cocitation analysis. Journal of the American Society for Information Science, 41, 453-458.
Cyert, R. \& March, J. (1963). A behavioral theory of the firm. O xford: Blackwell.

Dierickx, I. \& Cool, K. (1989). Asset stock accumulation and sustainability of competitive advantage.Management Science, 35, 1504-1511.

Dimaggio, P. \& Powell, W. (1983). The iron cage revisited: Institutional isomorphism and collective rationality in organizational fields. American Sociological Review, 48, 147-160.

Diodato, V. (1994).Dictionary of bibliometrics. Binghamton: Haworth Press, 1994.

Dyer, J. \& Singh, H. (1998). The relational view: Cooperative strategy and sources of interorganizational competitive advantage. Academy of Management Review, 23, 660-679.

Eisenhardt, K. \& Martin, J. (2000). Dynamic capabilities: What are they? Strategic Management Journal, 21, 1105-1121.

Eisenhardt, K. (1989). Building theories from case study research. Academy of Management Review, $14,532-550$.

Eisenhardt, M. (1989). Making Fast Strategic Decisions in High-Velocity Environments. Academy of Management Journal, 32: 543-576.

Eisenhardt, K. (1988). Agency and institutional theory explanations, the case of retail salecompensation. Academy of Management Journal, 31, 488-511.

Filardi, F., Barros, F. D., \& Fischmann, A. A. (2014). Do homo empreendedor ao empreendedor contemporâneo: Evolução das características empreendedoras de 1848 a 2014. Revista IberoAmericada de Estratégia, 13(3), 123-140.

Fosfuri, A., Lanzolla, G., \& Suarez, F. (2013). Entry timing strategies: the road ahead. Long Range Planning, 46(4-5), 300-311.

Grant, M. (1996). Toward a Knowledge-Based Theory of the Firm. Strategic Management Journal, 17, $109-122$

Greene, H. (2003). Econometric Analysis, Saddle River, NJ: Prentice Hall.

Guedes, V. \& Borschiver, S. (2005). Bibliometria: Uma ferramenta estatística para a gestão da informação e do conhecimento, em sistemas de informação, de comunicação e de avaliação 
A Pesquisa em Administração Estratégica: Um Estudo Bibliométrico em Periódicos Internacionais de Estratégia no Período de 2008 a 2013

científica e tecnológica. Artigo apresentado no Encontro nacional de ciências da informação 2005, Salvador/BA, Brasil.

Hambrick, D. \& Mason, P. (1984). Upper echelons: The organization as a reflection of its top managers. Academy of Management Review, 9, 193-206.

Hannan, M. \& Freeman, J. (1984). Structural inertia and organizational change. American Sociological Review, 49, 149-64.

Helfat, C. E. \& Peteraf, M. A. (2009). Understanding dynamic capabilities: Progress along adevelopmental path. Strategic Organization, 7, 91-102.

Helfat, E., Finkelstein, S., Mitchell, W., Peteraf, A., Singh, H., Teece, D. J. \& Winter, G. (2007). Dynamic capabilities. Understanding Strategic Change in Organizations. Oxford: Blackwell Publishing.

Helfat, E. \& Peteraf, A. (2003). The Dynamic Resource-Based View: Capability Lifecycles. Strategic Management Journal, 24, 997-1010.

Henderson, R. \& Clark, K. (1990). Architectural Innovation: The reconfiguration of existing product technologies and the failure of establishment firms. Administrative Science Quarterly, 35, 9-30.

Hoffman, D. \& Holbrook, M. (1993). The intellectual structure of consumer research: A bibliometric study of author cocitations in the first 15 years of the Journal of Consumer Research. Journal of Consumer Research, 19, 505-517.

Hoskisson, R., Hitt, M., Wan, W. \& Yiu, D. (1999). Theory and research in strategic management: Swing of a pendulum. Journal of Management, 25, 417-456.

Jensen, M. \& Meckling, W. (1976). Theory of the firm: Managerial behavior, agency costs and ownership structure. Journal of Financial Economics, 3, 305-360.

Katila, R \& Ahuja, G. (1992). Something old, something new: A longitudinal study of search behavior and new product introduction. Academy of Management Journal, 45, 11831194.

Kogut, B. \& Zander, U. (1992). Knowledge of the firm, combinative capabilities, and the replication of technology. Organization Science, 3, 383-397.

Leonardbarton, D. (1992). Core Capabilities and Core Rigidities: A paradox in managing new product development. Strategic Management Journal, 13:111-125.

Levinthal, D. \& March, J. (1993).The myopia of learning. Strategic Management Journal, 14, 95112.

Levitt, B. \& March J. (1988). Organizational learning. Annual Review of Sociology, 14, 319-340.

Lieberman, B. \& Montgomery, B. (1988). 1St-mover advantages. Strategic Management Journal, 9, 41-58.

Lin, T. \& Cheng, Y (2010). Exploring the knowledge network of strategic alliance research: A cocitation analysis. International Journal of Electronic Business Management, 8, 152-160.

Lin, H., Su, J. \& Higgins, A. (2016). How dynamic capabilities affect adoption of management innovations. Journal of Business Research, 69, 862-876.

March, James G. \& Simon, H. (1958). Organizations. Wiley: New York, 1958.

March, J. (1991). Exploration and exploitation in organizational learning. Organization Science, 2, 71-87.

Martinkenaite, I. \& Breunig, K. (2016). The emergence of absorptive capacity through micro-macro level interactions, Journal of Business Research, 69, 700-708.

Nag, R.,Hambrick, D. \& Chen, M-J. (2007). What is strategic management, really? Inductive derivation of a consensus definition of the field. Strategic Management Journal, 28, 935-955.

Nelson, R. \& Winter, S. (1982). An evolutionary theory of economic change. Cambridge: Harvard University Press.

Nerur, S., Rasheed, A. \& Natarajan, V. (2008). The intellectual structure of the strategic management field: an author co-citation analysis. Strategic Management Journal, 29, 319-336. 
A Pesquisa em Administração Estratégica: Um Estudo Bibliométrico em Periódicos Internacionais de Estratégia no Período de 2008 a 2013

Ocasio, W. (1997). Towards an attention-based view of the firm. Strategic Management Journal, 18,187-206.

Pfeffer, J. \& Salancik, G. (1978). The external control of organizations: A resource dependence perspective. New York: Harper and Row.

Penrose, E. (1959). The theory of the growth of the firm. New York: John Wiley.

Peteraf, M. (1993).The Cornerstones of Competitive Advantage: A Resource-Based View. Strategic Management Journal, 14, 179-91.

Pilkington, A. \& Meredith, J. (2009).The evolution of the intellectual structure of operation management 1980-2006: A citation/co-citation analysis. Journal of Operations Management, $27,185-202$.

Porter, M. (1980).Competitive strategy: Techniques for analyzing industries and competitors. New York: Free Press.

Porter M. (1985). Competitive Advantage. Free Press: New York.

Powell, W. Koput, K. \& Smith-Doerr, L. (1996). Interorganizational collaboration and the locus of innovation: Networks of learning in biotechnology. Administrative Science Quartery, 41, 116-145.

Ramos-Rodriguez, A. \& Ruiz-Navarro, J. (2004). Changes in the intellectual structure of strategic management research: A bibliometric study of the Strategic Management Journal, 1980-2000. Strategic Management Journal, 25, 981-1004.

Schneider, J. \& Borlund, P. (2004). Introduction to bibliometrics for construction and maintenance of thesauri: Methodical considerations. Journal of Documentation, 60, 524-549.

Serra, F., Ferreira, M., Almeida, M. \& Vans, S. (2012). A pesquisa em administração estratégica nos primeiros anos do século XXI: Um estudo bibliométrico decitação e cocitação no Strategic Management Journal entre 2001 e 2007. Revista Eletrônica de Estratégia \& Negócios, 5, 257274.

Shafique, M. (2013). Thinking inside the box? Intellectual structure of the knowledge base of innovation research: 1988-2008. Strategic Management Journal, 34:62-93.
Sirmon, G.,Hitt, A. \& Ireland, D. (2007). Managing firm resources in dynamic environments to create value: Looking inside the black box. Academy of Management Review, 32, 273-292.

Small, H. \& Garfield, E. (1985). The geography of science: Disciplinary and national mappings. Journal of Information Science, 11,147-159.

Stinchcombe, L. (1965). Social Structure and Organizations. In: J. G. March (Ed.), Handbook, of Organizations (pp: 142-193). Chicago, IL: Rand McNally \& Company.

Stuart, E., Hoang, H. \& Hybels, C. (1999). Interorganizational endorsements and the performance of entrepreneurial ventures. Administrative Science Quarterly, 44, 315-349.

Sun, Y., \& Grimes, S. (2016). The emerging dynamic structure of national innovation studies: a bibliometric analysis. Scientometrics, 106, 1740 .

Szulanski, G. (1996). Exploring internal stickiness: Impediments to the transfer of best practice within the firm. Strategic Management Journal, $17,27-43$.

Tahai, A. \& Meyer, M. (1999).A revealed preference study of management journals direct influences. Strategic Management Journal, 20, 279-296.

Thompson, J. (1967). Organizations in action: Social science bases of administrative theory. New York: McGraw-Hill.

Thomson Reuters. (2013). Web of Science Factsheet. Disponível em: http://thomsonreuters.com/content/science/pdf/ Web_of_Science_factsheet.pdf Acesso em 02 de abril de 2013.

Teece, D. (1986). Profiting from technological innovation: Implications for integration, collaboration, licensing and public policy. Research Policy, 15, 285-305.

Teece, D., Pisano, G. \& Shuen, A. (1997). Dynamic capabilities and strategic management. Strategic Management Journal, 1 (8), 509-533.

Teece, J. (2007). Explicating dynamic capabilities: The nature and microfoundations of (sustainable) enterprise performance. Strategic Management Journal, 28, 1319-1350. 
A Pesquisa em Administração Estratégica: Um Estudo Bibliométrico em Periódicos Internacionais de Estratégia no Período de 2008 a 2013

Terjesen, S., Hessels, J., \& Li, D. (2016). Comparative international entrepreneurship: a review and research agenda. Journal of Management, 41(1), 299-344.

Tushman M. \& Anderson, P. (1986). Technological discontinuities and organizational environments. Administrative Science Quarterly, 31, 439-465.

Vasconcelos, F. \& Cyrino, A. (2000). Vantagem competitiva: os modelos teóricos atuais e a convergência entre a estratégia e a teoria organizacional. Revista de Administração de Empresas, 40, 20-37.

Vieira, V., Machado, R. K., Brey, N. K., \& Rosseto, C. R. (2012). Evidências das pesquisas que abordam a tipologia de Miles e Snow no Brasil. Revista Ibero-Americana de Estratégia, 11(2), 70-90.

Vogel, R. \& Güttel, W. (2013). The Dynamic Capability View in Strategic Management: A Bibliometric Review. International Journal of Management Reviews, 15, 426-446.

Wernerfelt, B. (1984). The resource-based view of the firm. Strategic Management Journal, 5, 171180.
White, G., Guldiken, O., Hemphill, T., Wu, H. \& Khoobdeh, M. (2016). Trends in international strategic management research from 2000 to 2013: Text miningand bibliometric analyses. Management International Review, 56, 35-65.

White, D. \& Mccain, K. (1998). Visualizing a discipline: An author co-citation analysis of information science, 1972-1995. Journal of the American Society for Information Science, 49, 327-355.

Williamson, O. (1985). The economic institutions of capitalism: Firms, markets, relational contracting. New York: The Free Press.

Williamson, O. (1975). Markets and hierarchies, New York: Free Press, 1975.

Zhang, Y. \& Liao, Z. (2015). Consequences of abusive supervision: A meta-analytic review. Asia Pacific Journal of Management, 32(4), 959-987.

Zollo, M. \& Winter, G. (2002). Deliberate learning and the evolution of dynamic capabilities. Organization Science, 13, 339-351.

Zott, C., \& Amit, R. (2013). The business model: a theoretically anchored robust construct for strategic analysis. Strategic Organization, 11(4), 403-411. 